\title{
Clinical Outcome of Pars Plana Vitrectomy With Or Without Intravitreal Bevacizumab As A Pre-treatment in Advanced Diabetic Eye Disease
}

\author{
Ambreen Gul ${ }^{1}$, Sairam Ahmed ${ }^{2}$, Fuad Ahmad Khan Niazi ${ }^{3}$, Ali Raza ${ }^{4}$ \\ ${ }^{1-4}$ Holy Family Hospital, Rawalpindi Medical University, Rawalpindi
}

\begin{abstract}
Purpose: To find out the clinical outcome of Pars Plana Vitrectomy with and without Intravitreal Bevacizumab as a pretreatment in advanced diabetic eye disease.

Place and Duration of Study: Ophthalmology Department, Holy Family Hospital Rawalpindi, from January 2018 to December 2018.

Methods: Sixty patients with advanced proliferative diabetic retinopathy were included. Patients were divided into two groups. In group A, patients had pars plana vitrectomy with pre procedure injection of intravitreal Bevacizumab and group B had vitrectomy without pre procedure intravitreal Bevacizumab. Amount of bleeding during vitrectomy, surgical time and rate of iatrogenic tears were noted in both groups. Outcome measures were post operative best corrected visual acuity at 6 months and post operative recurrent vitreous hemorrhage.

Results: Patients had a mean age of $63.83 \pm 7.314$ years. In group A, mild bleeding was seen in $33 \%$, moderate in $6.7 \%$ and severe in $0 \%$. In group B, mild bleeding was seen in $13.3 \%$, moderate in $46.7 \%$, and severe bleeding in $40 \%$. After 6 months, 27 (90\%) patients in group A showed improvement in best corrected visual acuity while it was seen in $12(40 \%)$ patients in group B. latrogenic tears were seen in $10 \%$ in group A and $36 \%$ in group B. Mean time of surgery in group A was $59.27 \pm 6.823$ minutes and in group B was $77.87 \pm 9.637$ minutes. Rate of recurrent vitreous hemorrhage after vitrectomy was $6.7 \%$ in group $A$ and $40 \%$ in group $B$.
\end{abstract}

Conclusion: Intravitreal injection of Bevacizumab is helpful in reducing surgical time and also decreases intraoperative and post operative bleeding.

Key Words: Bevacizumab, Vitrectomy, Proliferative Diabetic Retinopathy.

How to Cite this Article: Gul A, Ahmed S, Niazi FAK, Raza A. Clinical Outcome of Pars Plana Vitrectomy With or Without Intravitreal Bevacizumab As A Pretreatment in Advanced Diabetic Eye Disease. Pak J Ophthalmol. 2020; 36 (4): 341-347.

Doi: https://doi.org/10.36351/pjo.v36i4.1036

\section{INTRODUCTION}

Diabetes mellitus is a metabolic disease. ${ }^{1}$ In Islamic republic of Pakistan, prevalence of Diabetes is

Correspondence: Ambreen Gul

Holy Family Hospital

Rawalpindi Medical University, Rawalpindi

Email:amber-gul@hotmail.com

Received: April 4, 2020

Accepted: July 21, 2020 reported to be nearly $9 \%$ especially in individuals in third decade of life. ${ }^{2}$ Diabetic retinopathy is amongst the foremost reasons of visual deterioration in $2^{\text {nd }}$ to $6^{\text {th }}$ decade. ${ }^{3}$ According to different studies, the incidence of diabetic retinopathy is approximated to be $15.3 \%$ to $28.9 \%{ }^{4}$ In a study in Gaddap, a prevalence of $1.74 \%$ of advanced manifestations of proliferative diabetic retinopathy was seen. ${ }^{2}$ The risk of developing retinopathy is proportional to the time period of diabetes. Majority of type 1 diabetic individuals and $60 \%$ of type 2 diabetics develop retinopathy following 
a time period of $15-20$ years. $^{5}$ Dense non clearing vitreous hemorrhage $(\mathrm{VH})$ and tractional retinal detachment (TRD) are the outcomes of advanced diabetic eye disease (ADED). ${ }^{6,7}$ Vitreous gel is an avascular structure and VH develops due to leakage of blood into vitreous from damaged vessels. Causes of VH include proliferative diabetic retinopathy, retinal tear, retinal vascular disorders, posterior vitreous detachment and eye trauma. ${ }^{8} \mathrm{VH}$ that obscures fundus view, is generally caused by a retinal tear or retinal vasculopathy posing a typical clinical dilemma. ${ }^{9}$ Vasculopathy in diabetes results in retinal ischemia and sequentially, release of vascular endothelial growth factor (VEGF). VEGF expression is the core reason for retinal neo-vessels. These pathophysiological changes provide the ground for employment of anti-VEGF therapies in retinal neovascularization diseases. ${ }^{10}$ Bevacizumab (Avastin) is an effective anti-VEGF. It is a recombinant humanized monoclonal antibody effective for all forms of VEGF. Its anti-angiogenic potential efficiently reduces neo-vessels on iris and retina in proliferative stages and is also useful in the treatment of advanced diabetic eye disease. It also treats intraocular hemorrhages by stimulating retinal neovessels degeneration. ${ }^{11}$

Pars Plana Vitrectomy (PPV) was started in 1970 for vitreous hemorrhage due to proliferative diabetic retinopathy. ${ }^{12}$ PPV plays a vital role in treating complicated ADED. TRD is outlined as tractional bands causing retinal elevation either clinically or spotted on B-scan ultrasound signifying a principal problem. Non-resolving VH is defined as bleed in the vitreous cavity that is constant (for a minimum of 3 months) or is recurrent. ${ }^{13}$ The aim of PPV in advanced diabetic eye disease is to refurbish vision by clearing blood in vitreous cavity and removing proliferative tractional fibrovascular membranes. It is also aimed to alleviate the retinal neovascularization by retinal endo laser photocoagulation. ${ }^{14}$ Key alarms in vitrectomy for complicated proliferative diabetic retinopathy are surgical complications like prolonged surgical time due to intra-operative bleeding, iatrogenic retinal tears, post-operative recurrent vitreous hemorrhage and neovascular glaucoma. They result in poor visual and anatomical outcomes. ${ }^{15}$

Currently, surgical outcomes of intravitreal Bevacizumab (IVB) as an add-on to PPV in managing diabetic eye disease have been unfolded in several trials. Hence, it was proposed that administration of
IVB preoperatively can be beneficial to decrease per operative bleeding in ADED. The utilization of antiVEGF agents few days before diabetic vitrectomy also ends up in better improvement in postoperative best corrected visual acuity (BCVA), and reduces frequency of recurrent $\mathrm{VH}$. Recurring vitreous hemorrhage is a frequent cause for reoperation. In majority of cases, recurrent hemorrhage in vitreous cavity is reported at some point within the initial six months postoperatively but may occur long duration afterward. ${ }^{16,17}$ However, its use remains controversial as some studies have mentioned a rapid progression of tractional detachment after IVB. ${ }^{18}$ Rationale of this study was to relinquish ample information regarding effectiveness of Avastin injection given as an intravitreal adjunct prior to PPV for the surgical management of proliferative diabetic retinopathy.

This prospective study was conducted to scrutinize, explore and compare the effect and success of IVB as an add-on in patients planned for three port PPV with ADED.

\section{METHODS}

After approval by hospital ethical review committee, the study was conducted at Department of Ophthalmology, Holy Family Hospital, Rawalpindi Medical University from January to December 2018. Sample size was drawn from the software by taking the statistical conditions of $95 \%$ confidence interval with 5\% margin of error. Sample included sixty eyes of sixty patients of either gender aging between 45 75 years with advanced diabetic ocular disease. The patients underwent pars plana vitrectomy. Only those patients who had TRD threatening macula, TRD with $\mathrm{VH}$ or non-clearing $\mathrm{VH}$ of a minimum of three months duration having good metabolic and hypertensive control were included in the study. Patients who had vitreoretinal or any intraocular surgery within 6 months or who had received Avastin within 6 weeks were excluded. We also excluded patients with known coagulation abnormalities or on anticoagulants. Patients were enrolled through DR (Diabetic retinopathy) clinic, Department of Ophthalmology Holy Family Hospital. Detailed informed consent was sought from all patients. Self-designed proforma was used to enter data. Total 60 patients were divided into two groups; group had 30 cases. Patients of group A underwent PPV with IVB of $1.25 \mathrm{mg} / 0.05 \mathrm{ml}, 1$ week (7 days) before surgery. Group B had 23 gauge 
vitrectomy with no intravitreal Avastin injection preoperatively. These patients were operated by single experienced vitreoretinal surgeon. Data including patient demographics, duration and category of diabetes (type 1 or 2 ) were recorded. Every patient underwent detailed ophthalmic examination including best-corrected visual acuity (BCVA) with Snellen decimal chart and slit lamp bio-microscopy and fundoscopy with $90 \mathrm{D}$ lens. Fundus images were taken by non-mydriatic fundus camera. Intra-ocular pressure (IOP) was taken by GAT goldman applanation tonometer. B-scan ultrasonography was done preoperatively to find out the condition of retina in cases of intense $\mathrm{VH}$.

A $10 \%$ povidone-iodine swab stick was applied to coat the injection site. After sterilized preparation, bevacizumab (Avastin) in dosage of $1.25 \mathrm{mg} / 0.05 \mathrm{ml}$ was injected intravitreally in the operation theatre under topical proparacaine drops. Injection site was marked with calipers at $4 \mathrm{~mm}$ in phakic and $3.5 \mathrm{~mm}$ in pseudophakic eyes, posterior to the limbus. Prophylactic topical antibiotic (Moxifloxacin) was started one day before the procedure. Drops were continued for a week after Avastin injection. Group A patients had PPV one week after injection by the same consultant surgeon under local anaesthesia. All vitrectomies were done using Feros Vitrectomy machine (Oertli, Switzerland).

After local anesthesia, surgical scrubbing and draping was done. Conjunctival peritomy was done 360 degrees. Sclerotomy ports were made using 23gauge micro-vitreoretinal knife. Core vitrectomy was carried out employing a vitreous cutter of high velocity (cut rate of 3000 cycles/minute). After intravitreal triamcinolone acetonide injection, residual vitreous was visualized along with epiretinal and vitreoretinal proliferation adhesions. Tangential and anterior-posterior traction was alleviated by cutting the peripheral vitreous. All tangential traction including proliferative epiretinal fibrous membrane was relieved as much as possible by segmentation, dissection and delamination with the help of vitreoretinal endo forceps and scissors. Scleral depression was done to aid 360 degree peripheral vitreous gel removal by shaving. A binocular indirect ophthalmo-microscope (BIOM) was employed in all cases consequently after adequate clearance of the vitreous hemorrhage to permit revelation of the posterior pole when required. High-speed 23-gauge cutter (up to $2500 \mathrm{cuts} / \mathrm{min}$ ) was accustomed to cut membranes very near to the retina.
No ILM peeling was done. With cautious lifting of the posterior hyaloid and indenting the periphery with muscle hook, scrupulous shaving of the vitreous base was done to eliminate the maximum amount of remaining blood as possible. Full attention was paid to alleviate traction. Panretinal photocoagulation was done with endolaser. Silicone oil $5000 \mathrm{cst}$ was used for internal tamponade. All ports of sclera and peritomy incisions were closed with 6/0 vicryl. Sub-conjunctival injection of steroid-antibiotic was given and eye pad was applied. One week face down posturing was done in all cases that had undergone air/fluid exchange. Hemostasis was maintained by raising ocular infusion pressure or by administering endo-diathermy. Amount of bleeding during vitrectomy surgery was observed by trained assistant and it was categorized as mild, moderate, severe or none. No bleeding, if none occurred during surgery, mild if bleed was stopped by increasing the ocular perfusion pressure, moderate if bleeding was stopped by increasing IOP for longer time, and severe, if endodiathermy was necessitated. Both groups A and B were compared for total bleed during surgery. None to mild bleeding cases were put in the category of successful response to IVB treatment. All patients were examined at $1^{\text {st }}, 7^{\text {th }}$, and $14^{\text {th }}$ day post operatively and followed up after every month till six months. Early postoperative VH was defined as vitreous bleed within a month post vitrectomy. Patients were followed up for at least 6 months.

Computer software package SPSS version 23.0 was used to analyze data. For quantitative variables like age, duration of diabetes, time of surgery; mean \pm S.D was calculated. For qualitative variables like gender, type of diabetes, bleeding during surgery, iatrogenic retinal tear and frequency (percentage) was calculated. Student t-test was used to compare statistical significance between two groups. $\mathrm{P}$ value less than 0.05 was considered statistically significant.

Primary clinical outcomes was flat retina postoperatively and visual outcome was BCVA at six months. Intraoperative complications including bleeding during surgery, necessity for endo-cautery to stop bleed, mean time of surgery and iatrogenic tears in retina were analyzed and compared in both groups.

\section{RESULTS}

There were 60 patients with 30 in each Group A and B. Mean age in group A was $63.73 \pm 8.387$ years and 
in group B was $63.83 \pm 7.314$ years. Among 60 patients there were $27(45 \%)$ males and $33(55 \%)$ females. There were 13 males and 17 females in group A and 14 males and 16 females in group B. Mean duration of diabetes was $17.50 \pm 5.111$ years in group A and $14.43 \pm 3.626$ in group B. In group A, (IDDM) insulin dependent diabetes mellitus was seen in 4 patients and (NIDDM) non insulin dependent diabetes mellitus was seen in 26 patients while in group B, IDDM was seen in 3 patients and NIDDM was seen in 27 patients. The difference between two groups was not statistically significant as regards to demographic details (age, gender, type and duration of diabetes) as $P$ value was more than 0.05 .

Intraoperative bleeding quantity was less in Group-A as compared to Group-B ( $\mathrm{p}$ value 0.001). Comparison between two groups for per operative bleeding is shown in Table 1.

Table 1: Intraoperative Bleeding in Avastin Group A and Control Group B.

\begin{tabular}{llrrrrr}
\hline & & \multicolumn{3}{c}{ Intraoperative Bleeding } & \multirow{2}{*}{ Total } \\
& & & & \\
None & Mild & Moderate Severe & \\
Group & A(Avastin group) & 18 & 10 & 2 & 0 & 30 \\
Total & B(control group) & 0 & 4 & 14 & 12 & 30 \\
\hline
\end{tabular}

The rate of iatrogenic tears in group A was $10 \%$ and in group B was $36 \%$ with a p value of 0.015 which was statistically significant. Mean time of surgery in group A was $59.27 \pm 6.823$ minutes and in group B was $77.87 \pm 9.637$ minutes. Meantime of surgery (mean \pm S.D) of Group-A was appreciably less in comparison to Group B $(\mathrm{p}=0.001)$.

In Group A, 28 (93.3\%) patients had no VH up to 6 months; whereas, in Group B, $18(60 \%)$ patients had no $\mathrm{VH}$ up to 6 months with asignificant p-value of 0.002 . The rate of recurrent bleed in vitreous cavity was $6.67 \%$ in group A and $40 \%$ in group B post operatively. There was statistically significant difference between two groups ( $p$ value 0.001). Postoperative rubeosis iridis was seen in $3.33 \%$ patient in group A as compared to $23.3 \%$ patients in group B $(\mathrm{p}=0.003)$.

Anatomical success was seen in $90 \%$ patients in group A and $60 \%$ of patients in group B $(p=0.001)$. In this study, visual outcome of BCVA at 6 months was measured in terms of stable, improved or worse visual acuity. At 6 months, 27 (90\%) cases in group A showed improved BCVA whereas in group B, 12 $(40 \%)$ cases had improved BCVA, p value of 0.001 . The results are shown in table 2 and figure 1 as under.

Table 2: Post operative BCVA in Avastin group $A$ and Control group $B$.

\begin{tabular}{llccc}
\hline \multicolumn{1}{c}{$\begin{array}{c}\text { Post Operative Best } \\
\text { Corrected VA }\end{array}$} & \multicolumn{2}{c}{$\begin{array}{c}\text { Group } \\
\text { Avastin } \\
\text { Group A }\end{array}$} & $\begin{array}{c}\text { Control } \\
\text { Group B }\end{array}$ & Total \\
\hline $\begin{array}{l}6 / 6 \text { to } 6 / 18 \\
(1.00-0.33)\end{array}$ & 5 & 1 & 6 \\
$\begin{array}{l}\text { Post Operative } \\
\text { BCVA }\end{array}$ & $\begin{array}{l}(0.24 \text { to } 6 / 60 \\
\text { less than 6/60 or } \\
\text { Counting fingers } \\
(0.08-0.03)\end{array}$ & 3 & 11 & 33 \\
Total & & 30 & 30 & 60 \\
\hline
\end{tabular}

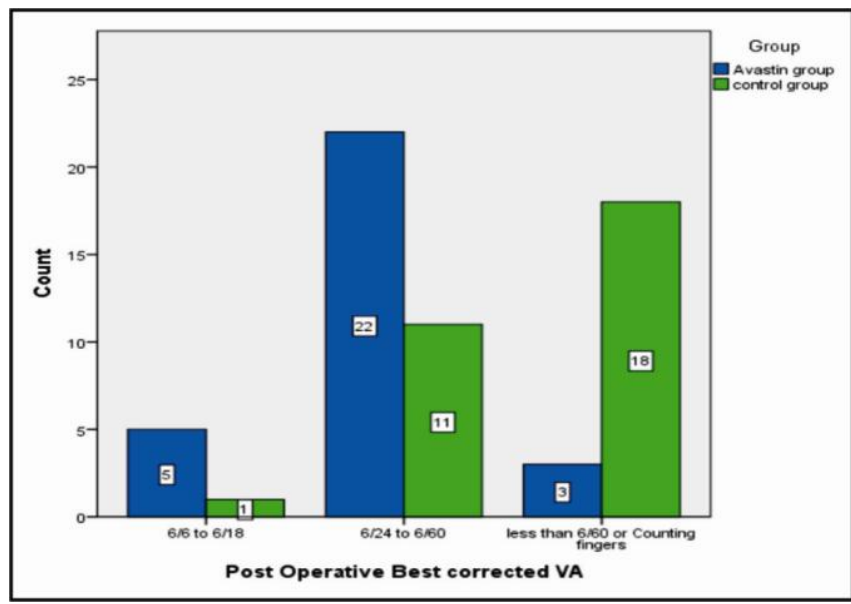

Fig. 1: Postoperative BCVA in Avastin Group A and Control Group B.

\section{DISCUSSION}

This study found that PPV with pre-operative use of IVB can be beneficial in reducing the risk of intraoperative hemorrhage, reducing the surgical time, decreasing the chance of postoperative hemorrhage and improving BCVA. In Pakistan, diabetic patients present in late complicated stages due to lack of understanding about complications of the disease, poor socioeconomic status of patients leading to poor acquiescence with medications which results in developing advanced form of diabetic eye disease.

In a study done by Rizzo et al patients had a mean age of 52 years. ${ }^{10}$ Yeoh et al reported a mean age of 46 years. ${ }^{19}$ Haseeb et al reported most of patients in the range of $56-65$ years $^{20}$ and in a study done by Faisal et al, age ranged from $47-65$ years with a mean of 
58.1 years. ${ }^{21}$ Different studies have shown different ages of patients with diabetic retinopathy. In our study, advanced disease was seen in sixth decade. Mean duration of disease of diabetes mellitus in our study was 17.50 years in Avastin group and 14.43 years in control group. Avery et al conducted a trial in which the mean duration of disease was 15 years. ${ }^{22}$ Prevalence of advanced diabetic eye disease increases as duration of diabetes increases. Faisal et al reported a comparatively shortermean duration of disease ${ }^{21}$, which can be attributed to poor compliance of patientsto medications leading to rapid progression of proliferative retinopathy.

In proliferative diabetic retinopathy, dissection of adherent proliferative fibrovascular membrane leads to repetitive intraoperative hemorrhage which obscures visibility and hence lengthening surgery time. Increased use of endo-diathermy for intraoperative bleeding also induces a high risk of inflammatory membrane and postoperative uveitis. Decreased circulation to the optic disc and corneal edema can be caused by elevated ocular perfusion pressure for prolonged duration to control intra operative hemorrhage. Pre-PPV intra-vitreal Avastin facilitates easy separation of proliferative fibrovascular membranes from retina with less intraoperative bleeding. This occurs as a result of degeneration of vascular complex of fibrovascular membranes aiding segmentation and dissection. Clear view during surgery reduces the duration of surgery. ${ }^{5,7,8,10}$.

Rizzo et al reported no intra-operative hemorrhage in $54 \%$ cases in group that received Avastin as compared to $18 \%$ in the group that had not received Avastin. ${ }^{10}$ In a similar study conducted by Faisal et al in non-Avastin group no bleeding was observed in only $7.1 \%$ and severe in $71.4 \%$. In Avastin group no bleeding was seen in $60.7 \%$ andsevere in only $17.9 \%$ of cases. ${ }^{21}$ El-Batarny reported severe bleeding in all 15 cases in non Avastin group. In group with pre vitrectomy Avastin, no bleeding was seen in $13.3 \%$ and severe in $26.6 \%$. $^{23}$

IVB also decreases post-PPV vitreous hemorrhage. Dissected and segmented fibrovascular complexes typically re-bleed in 7 days of surgery. Nevertheless, the cause of immediate post vitrectomy hemorrhage in vitreous cavity is generally hard to determine. In the current study, patients were examined postoperatively at 1 month, 3 months and 6 months for recurrent vitreous hemorrhage. The incidence of post vitrectomy recurrent hemorrhage in vitreous cavity in the Avastin group was significantly low. Haseeb et al reported no VH in Avastin group upto 6 months. ${ }^{20}$ Similar results have been reported by Cooper et al. ${ }^{24}$

In our study mean time of surgeryin group A was 59.27 mins \pm 6.82 and in group B was 77.87 mins \pm 9.63. Difference in mean time of surgery was quite evident between two groups. Rizzo et al., reported 57 minutes in group with Avastin and 83 minutes in nonAvastin group. ${ }^{10}$ Faisal et al reported $64 \pm 10.35$ minutes in Avastin group and $80.5 \pm 10.22$ minutes in non-Avastin group. ${ }^{21}$ In another study done by ElBatarny, mean surgical time was $93.3 \pm 11.6$ minutes in non-Avastin group as compared to $61.6 \pm 14.5$ minutes in Avastin group. ${ }^{23}$ The reasons for reduction in the operating time and facilitation of surgery were easier dissection of membranes owing to the lack of bleeding during surgery simplifying surgical technique with fewer exchanges of instruments and clear view.

Greater intraoperative bleeding hinders the surgical field, hence chances of inducing intraoperative iatrogenic retinal tears are also higher. Rizzo et al, reported no iatrogenic retinal tear in Avastin group and $36.3 \%$ in non-Avastin group. ${ }^{10}$ Faisal et al reported retinal tears in $7.1 \%$ in Avastin group as compared to $18.6 \%$ in non-Avastin group. ${ }^{21}$ El-Batarny reported increased frequency of iatrogenic retinal tears in Group given Avastin as compared to non-Avastin group. ${ }^{23}$ Although it is presumed that TRD increases in patients receiving Avastin, it can be reduced by giving IVB $2-7$ days before surgery.

In the current study, 27 (90\%) patients in group A had improvement in best corrected visual acuity in comparison to $12(40 \%)$ patients in group B at six months post operatively.This is comparable to study by Haseeb et al in which Avastin group had $70 \%$ patients who showed improved VA. ${ }^{20}$ El-Batarny reported improved visual acuityin $87 \%$ patients with IVB group. $^{23}$ Likewise, Ahmadieh had better best corrected visual acuity in Avastin group in contrast to group with no preoperative IVB. ${ }^{14}$ Iqbal et al reported improvement of vision in $82 \%$ patients, stablein $10 \%$, and worse in $8 \%$ in Avastin group. ${ }^{25}$ Primary retinal reattachment rate significantly differed between two groups as well (90\% in group A versus $60 \%$ in group B). In our study, postoperative rubeosisiridis or hyphaema was seen in very few $(3.33 \%)$ patients in group A compared to $23.3 \%$ patients in group B. ElBatarny also stated a similar results. ${ }^{23}$ Ischemic retina leads to agreater release of VEGF in vitreous cavity, 
whereas intravitreal bevacizumab leads to suppression of VEGF expression causing pharmacological involution of retinal and iris neovascularisation.

\section{CONCLUSION}

Intravitreal bevacizumab prior to PPV for advanced diabetic eye disease is a useful adjunct in offering success both anatomically and visually. This reduces intraoperative bleeding, resulting in less need for hemostatic techniques with fewer intraoperative complications and lesser risk of post operative vitreous hemorrhage and rubeosis iridis.

\section{Ethical Approval}

The study was approved by the Institutional review board/Ethical review board.

\section{Conflict of Interest}

Authors declared no conflict of interest.

\section{REFERENCES}

1. American diabetes association (ADA), position statement; standards of medical care in diabetes. Diabetes Care, 2013: 36 (Suppl1): S11-66.

2. Mahar PS, Awan MZ, Manzar N, Memon MS. Prevalence of type-II diabetes mellitus and diabetic retinopathy: the Gaddap study. J Coll Physicians Surg Pak. 2010; 20 (8): 528-532.

3. Cheung N, Mitchell P, Wong TY. Diabetic retinopathy. Lancet, 2010; 376: 124-136.

4. Shaikh A, Shaikh F, Shaikh ZA, Ahmed J. Prevalence of diabetic retinopathy and influence factors among newly diagnosed diabetics in rural and urban areas of Pakistan: Data analysis from the Pakistan National Blindness \& Visual Impairment Survey 2003. Pak J Med Sci. 2008; 24 (6): 774-779.

5. Gupta V, Arevalo JF. Surgical management of diabetic retinopathy. Middle East Afr J Ophthalmol 2013; 20: 283-292.

6. Kanski JJ. Clinical ophthalmology: a systematic approach. 6th Ed. Philadelphia: Elsevier Limited; 2015: 536.

7. Alagöz C, Yıldırım Y, Kocamaz M, Baz O, Çiçek U, Çelik B, et al. The Efficacy of Intravitreal Bevacizumab in Vitreous Haemorrhage of Diabetic Subjects. Turk J Ophthalmol. 2016; 46: 221-225.

8. Okoye O, Okonkwo O, Oderinlo O, Hassan K, Ijasan A. Bilateral concomitant intravitreal antivascular endothelial growth factor injection: Experience in a Nigerian tertiary private eye care facility. Niger $\mathbf{J}$ Clin Pract. 2016; 19: 544-548.
9. Tan HS, Mura M, Bijl HM. Early vitrectomy for vitreous haemorrhage associated with retinal tears. Am J Ophthalmol. 2010; 150: 529-533.

10. Rizzo S, Genovesi-Ebert F, Di Bartolo E, Vento A, Miniaci S, Williams G, et al. Injection of intravitreal bevacizumab (Avastin) as a preoperative adjunct before vitrectomy surgery in the treatment of severe proliferative diabetic retinopathy. Graefes Arch Clin Exp Ophtalmol. 2008; 246: 837-842.

11. Joob B, Wiwanikit V. Intravitreal Bevacizumab in Vitreous Hemorrhage and Diabetes Mellitus. Turk J Ophthalmol, 2017; 47 (2): 123-124.

12. Shi L, Huang YF. Postvitrectomy diabetic vitreous haemorrhage in proliferative diabetic retinopathy. J Res Med Sci. 2012; 17: 865-871.

13. Yang X, Xu J, Wang R, Mei Y, Lei H, Liu J, et al. A randomized controlled trial of conbercept pretreatment before vitrectomy in proliferative diabetic retinopathy. J Ophthalmol. 2016; 2016: 1-8.

14. Ahmadieh H, Shoeibi N, Entezari M, Monshizadeh R. Intravitreal bevacizumab for prevention of early postvitrectomy haemorrhage in diabetic patients: A randomized clinical trial. Ophthalmology, 2009; 116: 1943-1948.

15. Yeh PT, Yang CM, Lin YC, Chen MS, Yang CH. Bevacizumab pretreatment in vitrectomy with silicone oil for severe diabetic retinopathy. Retina 2009; 29:76874. Exp Ophtalmol. 2008; 246: 837-842.

16. Zhang ZH, Liu HY, Hernandez-Da Mota SE, Romano MR, Falavarjani KG, Ahmadieh H, et al. Vitrectomy with or without preoperative intravitreal bevacizumab for proliferative diabeticretinopathy: A meta-analysis of randomized controlled trials. Am J Ophthalmol. 2013; 156: 106-115.

17. Lo WR, Kim SJ, Aaberg TM Sr, Bergstrom C, Srivastava SK, Yan J, et al. Visual outcomes and incidence of recurrent vitreous haemorrhage after vitrectomy in diabetic eyes pretreated with bevacizumab (Avastin). Retina, 2009; 29: 926-931.

18. Jonas JB, Schmidbauer M, Rensch F. Progression of tractional retinal detachment following intravitreal bevacizumab. Acta Ophthalmol. 2009; 87: 571-572.

19. Yeoh J, Williams C, Allen P, Buttery R, Chiu D, Clark B, et al. Avastin as an adjunct to vitrectomy in the management of severe proliferative diabetic retinopathy: a prospective case series. Clin Exp Ophthalmol. 2008; 36: 449-454.

20. Haseeb U, Rehman AU, Haseeb M. Visual outcomes of pars plana vitrectomy alone or with intra vitreal bevacizumab J Coll Physicians Surg Pak, 2019; 29 (8): 728-731.

21. Faisal SM, Tahir MA, Cheema AM, Anjum MI. Pars plana vitrectomy in vitreous hemorrhage with or without Intravitreal Bevacizumab: A comparative overview. Pak J Med Sci. 2018; 34 (1): 221-225. 
22. Avery RL, Pearlman J, Pieramici DJ, Rabena MD, Castellarin AA, Nasir MA, et al. Intravitreal bevacizumab (Avastin) in the treatment of proliferative diabetic retinopathy. Ophthalmology, 2006; 113 (10): 1695.

23. El-Batarny AM. Intravitreal bevacizumab as an adjunctive therapy before diabetic vitrectomy. Clin Ophthalmol. 2008; 2 (4): 709-716.

24. Cooper B, Shah GK, Grand MG, Bakal J, Sharma S. Visual outcomes and complications after multiple vitrectomies for diabetic vitreous haemorrhage. Retina, 2004; 24: 19-22.

25. Iqbal A, Orakzai OK, Khan MT, Sanaullah J. Visual outcome after pars plana vitrectomy in diabetic vitreous haemorrhage. J Pak Med Inst. 2016; 30: 23-9.

\section{Authors' Designation and Contribution}

Ambreen Gul; Senior Registrar: Preparing the manuscript, data collection, writing the article, protocols and designs to be followed in the study.

Sairam Ahmed; Resident: Data analysis, manuscript writing and review.

Fuad Ahmad Khan; Associate Professor: Data analysis, manuscript writing and review.

Ali Raza; Professor and Head of Department: Data analysis, manuscript writing and review. 